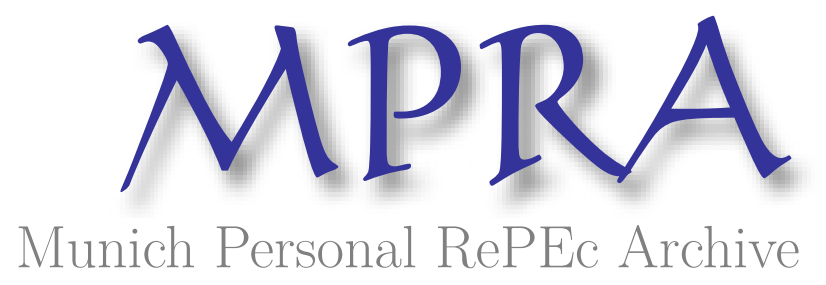

\title{
Financial exclusion and the cost of incomplete participation
}

Botti, Fabrizio and Bollino, Carlo Andrea

December 2012

Online at https://mpra.ub.uni-muenchen.de/44065/

MPRA Paper No. 44065, posted 31 Jan 2013 05:24 UTC 


\title{
Financial Exclusion and the Cost of Incomplete Participation
}

\author{
Carlo A. Bollino ${ }^{1}$, Fabrizio Botti ${ }^{2}$
}

\begin{abstract}
Economic and social implications of the access to financial services both in developed and in developing countries have increasingly promoted the debate around the issue of considering "financial inclusion" as a public good, according to potential positive externalities associated to greater financial participation. If the role of financial inclusion as a public good, and the enhanced efficiency of public policy following a greater participation in the financial markets are established in an abstract way, a numerical estimate of the potential costs of incomplete participation in the financial system is still not explicitly addressed in the literature. The study designs a simplified approach for the calculation of the cost of financial exclusion through the identification of a general functional form representing the cost of incomplete participation encountered by financial actors or by the public policy aimed at alleviating financial exclusion. Such a cost is estimated parametrically according to alternative subgroups of financial institutions with different levels of depth of outreach corresponding to distinct orientation toward individuals excluded from the mainstream financial sector. Calculations show the role of financial exclusion in generating inefficiencies that raise the cost of accessing to financial transactions for all the participating individuals, or, in a policy perspective, the cost to tackle incomplete participation.
\end{abstract}

\section{Introduction}

A general consensus recently aroused amongst European experts about financial exclusion being part of the wider concept of social exclusion and on its definition as "a process whereby people encounter difficulties accessing and/or using financial services and products in the mainstream market that are appropriate to their needs and enable them to lead a normal social life in the society which they belong" (European Commission 2008).

Economic and social implications of the access to financial services both in developed and in developing countries have increasingly promoted the debate around the issue of considering "financial inclusion" as a public good. Microfinance advocates emphasize the nature of public good of the provision of income-generating services by Microfinance Institutions (MFIs) (Balkenhol, 2008). According to Merhotra et al. (2009) financial inclusion differs from typical public good as defence, but is deemed to be a "quasi public good" as it meets the conditions of non-rivalness in consumption and non-excludability to a large degree. Moreover, positive externalities are associated to financial inclusion as the advantages of network effects for participants, the expanding value of the overall financial system, and a more effective monetary policy resulting from a greater participation (Agrawal, 2008).

Financial inclusion also emerged in recent years as a key policy issue at the European level in the framework of the 2007 Single Market Review ${ }^{3}$ and of the following "Recommendations

\footnotetext{
${ }^{1}$ Dipartimento di Economia, Finanza e Statistica, Università degli Studi di Perugia, via A. Pascoli 20, 06123 Perugia, e-mail: bollino@unipg.it.

${ }^{2}$ Dipartimento di Economia, Finanza e Statistica, Università degli Studi di Perugia, via A. Pascoli 20, 06123 Perugia, e-mail: fabrizio.botti@unipg.it.
} 
on access to a basic payment account" (2011/442/EU) adopted by the European Commission in $2011^{4}$.

If the role of financial inclusion as a public good, and the enhanced efficiency of public policy following a greater participation in the financial markets are established in an abstract way, a numerical estimate of the potential costs of incomplete participation in the financial system is still not explicitly addressed in the literature.

A simplified approach for the calculation of the cost of financial exclusion is provided in the present study through the identification of a general functional form representing the cost of inclusion as showed by Nordhaus (2009) for the case of the CO2 abatement cost in the framework of participation to International Protocols.

The aim of the study is to capture in an intuitive way the general effect of financial exclusion in an aggregate model presented in section 1. An empirical estimation of the convexity of the marginal cost of inclusion is carried out in section 2 using MIX Market data on 2,232 Microfinance Institutions (MFIs). In the last section, we present a preliminary application of the model of incomplete participation and compute the cost penalty factor associated to different degree of financial institutions commitment to inclusion.

\section{The model of incomplete participation}

A specific reduced functional form for the cost of financial intermediation is firstly assumed. In a policy perspective, the cost function is also deemed to capture the abatement cost of financial exclusion, and in case of full participation is the following:

$$
C=\left[\sum_{i=1}^{n} C_{i}\right]=\left[\sum_{i=1}^{n} Y_{i}\right] \mu^{\beta}
$$

In the above full inclusion cost function, $\mathrm{C}_{\mathrm{i}}$ is the average cost, $\mathrm{Y}_{\mathrm{i}}$ is the total output (value added inclusive of oligopoly profits), $\mu$ is the inclusion rate, and $\beta$ is a fixed parameter. The inclusion rate captures financial deepening (or depth of outreach improving) efforts undertaken by financial actors committed to those excluded from the mainstream financial markets (i.e. microfinance programmes or other specific actions to vulnerable categories of potential clients such as migrants, ethnic minorities, elderly people and women) and translates in cost inefficiencies for the proposing institution. Regardless of full inclusion, $\mu$ is positive

\footnotetext{
${ }^{3}$ The annex to the Single Market Review [SEC(2007)1520] required that "...reflection should progress on how to ensure that, by a certain date nobody is denied access to a basic bank account".

${ }^{4}$ The EC recognized that recognized "access to a bank account has become a pre-condition for participating fully in the economic and social life of a modern society".
} 
(even if low) on account of persistent financial market imperfections (e.g. informational asymmetries).

Then assume that only a fraction of financial actors provides products and services to the target groups of financially excluded individuals (henceforth low-end institutions), corresponding to a share of the overall financial output $\pi$. In such a financial exclusion scenario, define the inclusion rate of low-end institutions as $\mu^{P}$, and that of those aiming at individuals with full or partial access to financial markets as $\mu^{N P}=0$. The inclusion cost function of the former under these assumptions is the following:

$$
C^{P}=Y^{P}\left(\mu^{P}\right)^{\beta}
$$

where $Y^{P}$ is the output of low end institutions and the overall inclusion rate is given by:

$$
\mu=\mu^{P} \pi
$$

Recalling that $Y^{P}=\left[\sum_{i=1}^{n} Y_{i}\right] \pi$, the inclusion cost funtion with financial exclusion and partial commitment of financial institutions to outreach improving efforts is the following:

$$
C^{P}=\left[\sum_{i=1}^{n} Y_{i}\right] \pi(\mu / \pi)^{\beta}
$$

or

$$
C^{P}=\left[\sum_{i=1}^{n} Y_{i}\right] \mu^{\beta} \pi^{1-\beta}
$$

The inclusion cost function (5) thus shows the effects of financial exclusion on intermediaries and public policy aimed at alleviating financial exclusion. If compared to the full inclusion cost function (1), it exhibits a cost penalty factor $\pi^{1-\beta}$ accounting for the extra cost encountered by financial actors involved in outreach improving efforts. Therefore, financial exclusion generates inefficiency for low-end institutions represented by the exponential function of the parameter $(\beta-1)$ corresponding to the convexity of the marginal cost of inclusion. 


\section{The empirical estimation of the marginal cost of financial inclusion}

Any estimates of the cost of excluding a fraction of the overall population from financial markets and of the resulting cost inefficiency for low-end institutions rely on the shape of the inclusion cost function.

A comparison of equation (1) and (5) allows to identify a measure of the cost associated with financial exclusion and with algebraic manipulation it is easy to show that partial involvement of financial institutions in outreach improving programs raises total costs by a penalty factor $\pi^{1-\beta}$.

The inefficiency produced by financial exclusion is for low-end institutions an exponential function of the parameter $(\beta-1)$ that is the convexity of the marginal cost of inclusion. If marginal costs are constant, then $(\beta-1)$ equals zero and the cost-penalty factor is zero as well. Otherwise, if marginal costs increase with financial inclusion efforts, then the parameter $(\beta-1)$ $>0$ and financial exclusion is costly. If the marginal cost function is convex, inclusion costs would tend to accelerate with inclusion rate when financial institutions are partially committed to financially excluded individuals ${ }^{5}$.

We estimated the convexity parameter in the marginal cost of inclusion function using the 2012 Mix Market dataset on 2,232 Microfinance Institutions (MFIs) in 119 countries, both developing and developed.

A cross-sectional analysis of the Mix Market data has been undertaken for the year 2008, assuming the marginal cost of inclusion (MC) and the inclusion rate $(\mu)$ as proxied by respectively a cost efficiency measure (Operating Expenses over Assets or Loan Portfolio), and a standard indicator of the orientation of financial institutions towards the most disadvantaged individuals (Average Loan Balance per borrower over the Gross National Income per capita - ALB/GNI). The former is assumed to be a proxy of the operative costs (selection, screening, transaction costs) experienced by financial actors serving those excluded from the mainstream financial markets. The ALB/GNI is a commonly adopted indicator of financial institutions depth of outreach, especially in microfinance circles. Figure 1 shows the estimated $\log$ of the marginal cost function convexity.

$$
\ln \left(\mathrm{MC}_{\mathrm{i}}\right)=\beta_{0}+\beta_{1}\left(\mu_{\mathrm{i}}\right)+\beta_{2}\left(e f f_{i}\right)+\beta_{3}\left(\operatorname{prod}_{i}\right)+\beta_{4}\left(\operatorname{risk}_{i}\right)+\varepsilon_{\mathrm{i}}
$$

In order to account for the broad range of institutional and contextual characteristics of observed providers, we controlled for efficiency (average salary/GNI per capita), productivity (borrowers per loan officer) and risk (portfolio at risk $>30$ days/loan portfolio) variables.

\footnotetext{
${ }^{5}$ Nordhaus (2009) estimates a similar type of inefficiency cost induced by non participation of some countries to the Kyoto Protocol abatement policy.
} 
Fig.1 Estimated log of the marginal cost of inclusion

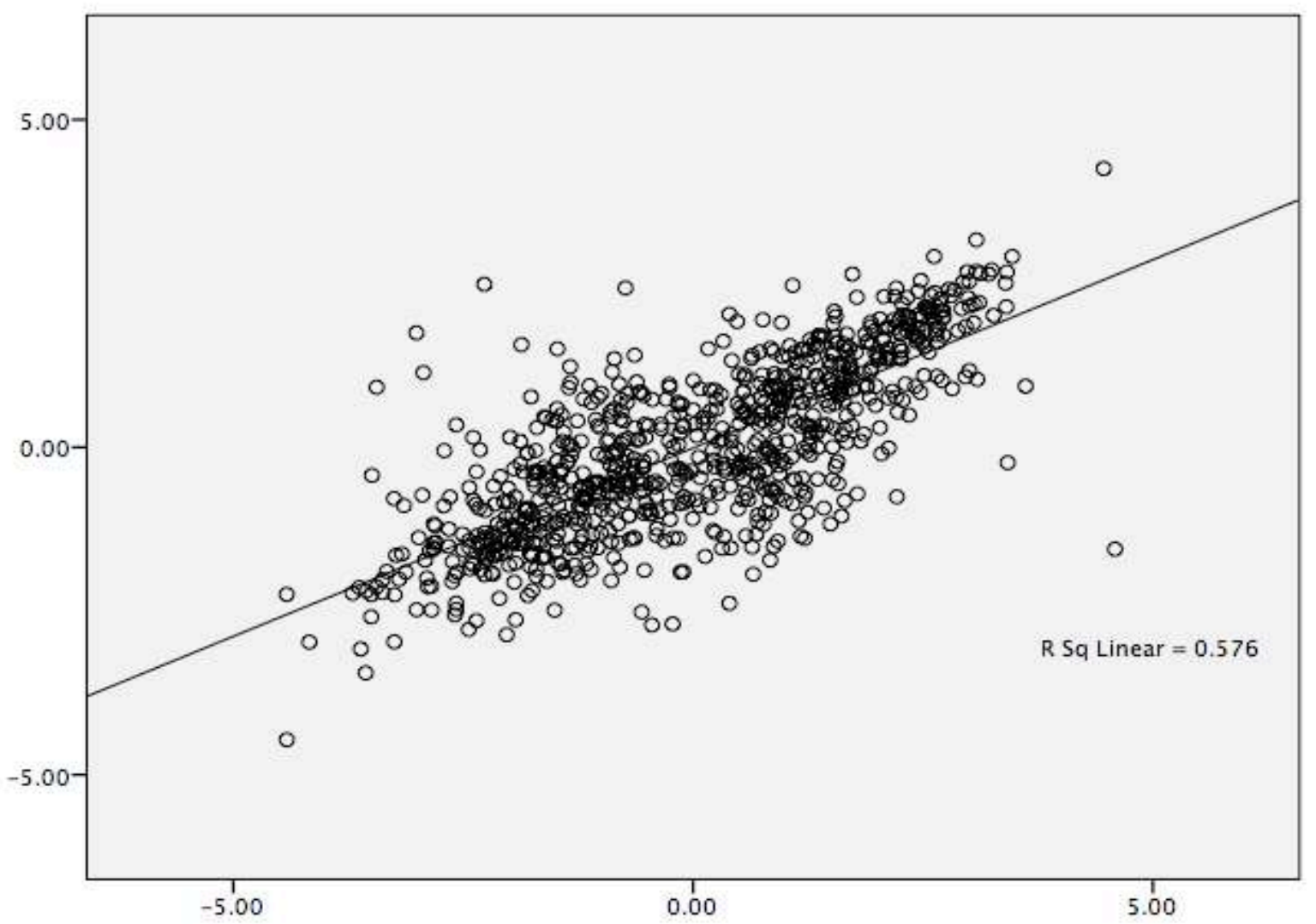

Source: Authors' calculation; MIX Market database, 2008

Estimates of the convexity parameters are summarized in Table 1 and range between 0.520 and 0.560 according to different proxies of the marginal cost of inclusion (operating expenses/assets and operating expenses/loan portfolio).

Table 1. Estimates of the convexity parameter in the marginal cost of inclusion

\begin{tabular}{lcc}
\hline & $(1)$ & $(2)$ \\
\hline$\beta_{0}$ & 16.771 & 17.470 \\
& $(.235)^{* *}$ & $(.255)^{* *}$ \\
\hline -(ALB/GNI p.c.) & .520 & .560 \\
& $(.015)^{* *}$ & $(.017)^{* *}$ \\
\hline Avg. Salary/GNI p.c. & .545 & .597 \\
& $(.021)^{* *}$ & $(.023)^{* *}$ \\
\hline Borrower/Loan officer & -.538 & -.581 \\
& $(.022)^{* *}$ & $(.024)^{* *}$ \\
\hline PAR30/Loan Ptf. & .037 & .062 \\
& $(.011)^{* *}$ & $(.012)^{* *}$ \\
\hline $\mathrm{R}^{2}$ & .646 & .642 \\
\hline Obs. & 847 & 847 \\
\hline
\end{tabular}

(1) Dependent Variable: Operational Expenses/Assets.

(2) Dependent Variable: Operational Expenses/Loan Ptf.

** Significant at $1 \%$

Standard errors in parentheses. 
In the next section we estimate the cost penalty factor associated to different degree of financial institutions commitment to inclusion by assuming that the exponent parameter is $\beta-1$ $=0.520$ owing to the better fit of the first model of Tab. 1 on available data.

\section{A preliminary application of the model of incomplete participation}

The inclusion cost function in section 1 ease to identify the cost inefficiencies arising from a low participation rate of financial institution in outreach improving efforts, given a certain degree of financial exclusion.

In our empirical estimation of the cost penalty factor of financial exclusion alleviating programmes, we assume that only financial actors with depth of outreach indicator below $20 \%$ or average low sizes lower than $\$ 150$ (those serving the low-end target market peer group) are actually providing access to individuals excluded from the mainstream financial market (Tab. 2).

Table 2. Financial Institutions by Target Market and Share of Gross Loan Portfolio (2008)

\begin{tabular}{|c|c|c|}
\hline Target market ${ }^{a}$ & $\begin{array}{c}\text { Share of Overall Gross Loan Portfolio } \\
(\%)\end{array}$ & $\begin{array}{c}\text { Cumulative share } \\
(\%)\end{array}$ \\
\hline Low end & 21.9 & 21.9 \\
\hline Broad & 57.7 & 79.5 \\
\hline High end & 10.1 & 89.6 \\
\hline Small Business & 10.4 & 100 \\
\hline
\end{tabular}

Supposing that the share of the overall gross loan portfolio belonging to low-end institutions reflects the portion of lending activity committed to financial exclusion alleviation $(\pi)$, the cost of inclusion is 2.20 times the cost under complete participation (Tab. 3). The inclusion cost function allows to estimate the impact of a greater involvement of financial actors with outreach improving efforts by computing the cost penalty factor associated to larger share of corresponding lending activity. A downscaling of financial actors currently with a depth of outreach between $20 \%$ and $149 \%$ (peer group of broad institutions) would almost halve the inclusion cost. 
Table 3. Cost of inclusion by financial institutions participation rate

\begin{tabular}{cc}
\hline Participation rate $(\pi)$ & Cost penalty factor $\left(\boldsymbol{\pi}^{\mathbf{1}-\boldsymbol{\beta}}\right)$ \\
\hline 21.9 & 2.20 \\
\hline 79.5 & 1.13 \\
\hline 89.6 & 1.06 \\
\hline 100 & 1 \\
\hline
\end{tabular}

Source: Authors' calculations; MIX Market database, 2008

These calculations show the role of financial exclusion in generating inefficiencies that raise the cost of accessing to financial transaction for all the participating individuals, or, in a policy perspective, the cost to tackle incomplete participation.

\section{Conclusion}

The inclusion of unbanked in the mainstream financial system has been increasingly recognized as a prerequisite for a full participation in economic and social life and a major concern in policy makers agenda at both the national and European level ${ }^{6}$. However, available evidence shows that a notable share of Europeans is still excluded from the access to even a basic payment account (European Commission, 2008). If the role of financial inclusion as a public good is commonly established in policy and academic circles, the achievement of a greater access to financial transaction is still a crucial challenge to attain an efficient policy.

The identification of a functional form for a inclusion cost function in order to attain a numerical estimation of the abatement cost of financial exclusion for intermediaries committed to individuals excluded from mainstream financial markets has been the major aim of the present study.

The participation cost function shows the inefficiency arising from financial exclusion for participants by identifying a cost penalty factor $\pi^{1-\beta}$. Considerable cost penalty from different participation rates are basically related to the convexity of the marginal cost function expressed by the exponential parameter $(\beta-1)$ that is higher than zero. This convexity implies that significant maximum benefits (i.e. low costs) can be achieved if a larger share of financial actors participates in outreach improving programmes. The reasoning above is quite

\footnotetext{
${ }^{6}$ See European Commission, "Recommendation on access to a basic payment account", C(2011) 4977/4.
} 
intuitive: if many groups do not participate, the penalty cost is high, because the efficiency benefits could hardly be achieved.

Numerical results in our study also highlighted the potential magnitude of the cost of financial exclusion actually sustained by the society and strengthen the call for policy actions to take coordinated efforts to promote greater access to financial services. 


\section{References}

Agrawal, A. (2008), "The need for financial inclusion with an Indian perspective", Economic Research, IDBI.

Balkenhol, B. (ed.) (2008), Microfinance and Public Policy: Outreach, Performance and Efficiency", Palgrave McMillan, 2007.

Carbo, S., E. Gardener, and P. Molyneux (2007), "Financial Exclusion in Europe", Public Money \& Management, 27(1), pp. 21-27.

Demirguc-Kunt, A., and and L. Klapper (2012), "Measuring Financial Inclusion: The Global Findex", The World Policy Research Observer, WP 6025.

Devlin, J. (2005), “A Detailed Study of Financial Exclusion in UK”, Journal of Consumer Policy, Vol. 28, n. 1, pp. 75-108.

European Commission (2008), "Financial services provision and prevention of financial exclusion”, Directorate-General for Employment, Social Affairs and Equal Opportunities, Inclusion, Social Policy Aspects of Migration, Streamlining of Social Policies.

Finney, A., and E. Kempson (2010), "Regression Analysis of the unbanked using the 200607 Family Resources Survey”, Personal Finance Research Center, University of Bristol.

Merhotra, N., V. Puhazhendhi, G. N. Gopakumaran, and B.B. Sahoo (2009). "Financial inclusion - an overview", Department of Economic Analysis and Research - National Bank for Agriculture and Rural Development, Mumbai.

Nordhaus, W. (2008), "The impact of treaty nonparticipation on the costs of slowing global warming", The Energy Journal, Vol. 30, Special Issue 2, pp. 39-51. 